\title{
Surveillance and Defensive Robo Security Guard
}

\author{
Parithosh Amin ${ }^{1}$, Pawan Kumar Shetty ${ }^{2}$, Shilpa K Vasudev ${ }^{3}$, Varsha Keshav Uchil ${ }^{4}$ \\ Student, ECE, SMVITM, Udupi, India ${ }^{1,2,3,4}$
}

\begin{abstract}
Now a day's robots are use in various applications like industries, building, military etc. for surveillance and defensive purpose. The robot patrols a particular area by live video streaming operated by the controller. For defensive purpose, whenever the intruder is detected, the gun is triggered by the controller. Here USB camera and gun is varied at any angle controlled by the controller. The sensors are used here to sense smoke at surroundings, metal detector sensors used to determine the presence of metal nearby and heat sensor used if there is any fire in the surroundings. The sensing unit when detected causes LED light to turn ON. The motion of robot is controlled by the DC motor using Arduino Bluetooth technology by an Android App. For live video streaming the Raspberry pi is interfaced with the USB camera.
\end{abstract}

Keywords: USB: - Universal Serial Bus, LED:-Light Emitting Diode, DC: - Direct Current, App:-Application.

\section{INTRODUCTION}

Robotic devices are used in all aspects of our world, it may be automation in factories, businesses, personal prosthetics, or used for off world exploration in space and on other planets. We rely on robotics every day to reduce the costs of products and services and to make our lives easier. The ability to control, modify and update these robotic devices remotely is crucial. This allows robots to go in places where humans may not. It also provides mobility to people with disabilities who must control them by other means. To develop truly useful robotic equipment, the devices must be designed in a manner that considers a functional, precise and simple interface.

The robot provides security guard service for office buildings, hospitals, stores, etc. to detect like fire, intruders, etc. If a sensor detects an emergency, this information is transmitted to the controller through a communication network. The controller can alert an emergency near the emergency site. Patrolling the premises is at type of security service. It is desirable to have a robot patrolling. It might be difficult to the worker to work in an environment, such as Welding is considered as a dangerous task for humans because of toxic gases emissions, Painting has similar problems to welding due to the use of toxic chemical products, assembly operation. Therefore a robot can replace human to do work.

The robot build by us can move from place to place across the ground. This robot can work in a human-centred space and cooperate with men by sharing a work-space together. The purpose of this project is to design and develop such an interface to provide control over a robotic guard from a remote location in a simulated environment applicable to realworld scenarios. Robot is implemented by interfacing the micro controller (Arduino) with microcomputer (Raspberry $\mathrm{Pi}$ ) by using serial port. The micro controller is programmed in Arduino $\mathrm{C}$ language. Raspberry is used as microcomputer which is connected to camera placed on top of the robot for live video streaming. This robot is having the gun below the camera so that the master can strike or destroy the enemies on his control. The movement of the chassis of the robot at the base and triggering of the gun is controlled by the controller through the mobile via Bluetooth using Arduino and Bluetooth module. The robots also detects if there is any fire in the place using heat sensor protecting that premises by sending alert information to the controller. If there is any human entering a particular premises the robot will view the human through USB camera. The controller gets alert regarding the intruder and he takes action. The action may be either he can capture the image for any necessary proof in future or he can take actions for the defensive purpose of the premises by using the gun. Here the human guards are replaced by this revolutionary robot as the guard to perform particular tasks.

\section{LITERATURE SURVEY}

"The robots systems is divided into four subsystems namely vision, manipulation, emergency sensing and communication" [1].

- Vision: The operator can understand the scene around the robot by remotely operating the camera vision system mounted on the robot.

- Manipulation: For dealing with emergencies, extinguishing small fires, the operator can operate the manipulation system remotely.

- Emergency sensing: The robot has sensor to detect heat, smoke, intruder, etc.

- Communication: Control data, vision data, wheel movement, etc. can be communicated between robot and operator. 


\section{IJIREEICE

'Autonomous Robot', a locomotion mechanism is designed and developed. This locomotion mechanism will form the chassis of an autonomous robot. Robot will be used in well structured engineered environments, on flat surfaces, used in various applications [2].

Comparing robots to humans "Vision is equal to camera, feeling is equal to sensors. Communication is equal to wires, fiber optics and radio, Brain is equal to computers and microprocessors "[3].

The most basic four-wheeled vehicle actually doesn't even use a differential. It has two wheels on each side that are coupled together and is steered just like differential steered tricycles. Since the wheels are in line on each side and do not turn when a corner is commanded, they slide as the vehicle turns. This sliding action gives this steering method its name as Skid Steer. Skid steered vehicles are a robust, simple design with good mobility, in spite of the inefficiency of the sliding wheels. Since the wheels don't turn, it is easy to attach them to chassis, and they don't take up the space required to turn [4].

\section{III.METHODOLOGY}

The main objective of this project is to control the robot wirelessly using USB camera for surveillance, gun triggering for defensive purpose and sensors like heat sensor, metal detector sensor, and smoke sensor used for detecting heat, metal and smoke at the surroundings.

Here the camera and gun can rotate for $360^{\circ}$ because of the rotation of upper part of the body of robot where gun and camera is placed. Even though the intruder is behind the robot, the robot can rotate its upper part of the body where the controller can view the intruder and can target him.

USB camera is interfaced with Raspberry pi for live video streaming shown in Fig. 1(b). Gun triggering and motion of the robot, rotation of upper part of the robot is controlled by the DC motor using Arduino interfaced with Bluetooth shown in Fig. 1(a). The sensors used here when detected makes the LED light ON, where these LEDs are placed in front of and slightly below the camera, so that only the colored light rays can be viewed in the camera and not the LED component. For smoke sensor blue LED is ON, whereas green for metal detector sensor and yellow for heat sensor as shown in Fig. 1(c).

\section{BLOCK DIAGRAM}

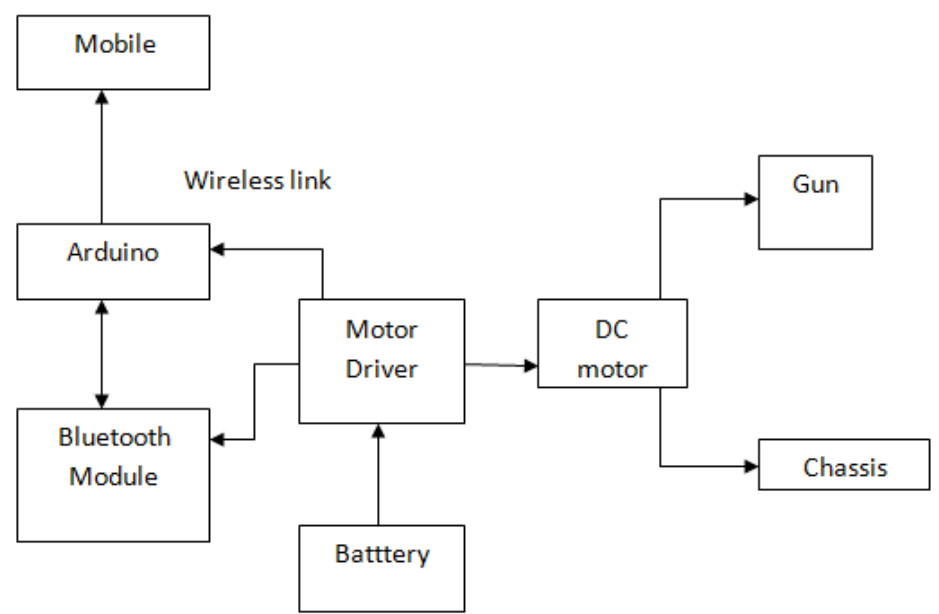

Fig. 1(a). Block diagram of proposed system results motion and rotation of upper part of robot and triggering of gun.

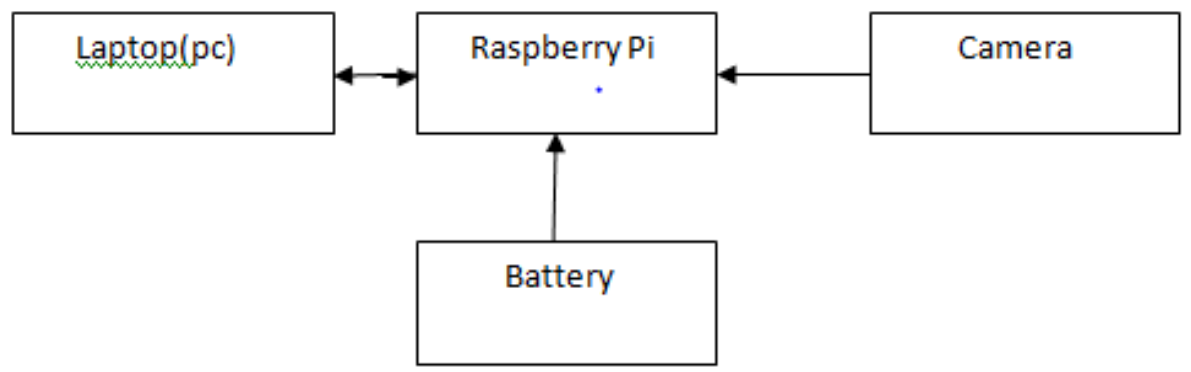

Fig. 1(b). Raspberry Pi interfaced with the camera. 
Vol. 5, Issue 6, June 2017



Fig. 1(c). Sensing unit viewed in camera by LED.

\section{HARDWARE DESCRIPTION RASPBERRY PI}

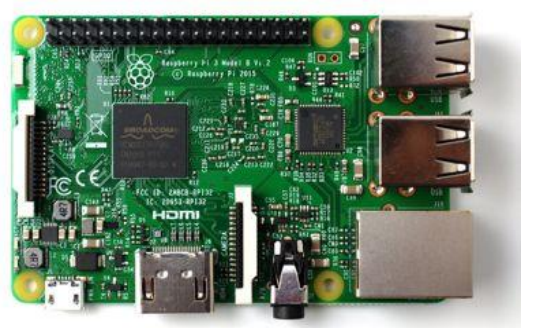

Raspberry Pi is a small computer about the size of a credit card performing various functions in many applications. The functionalities of Raspberry Pi components are

Micro USB power port:-The micro USB power port is used to power the Raspberry Pi device and provides 700mA at $5 \mathrm{~A}$.

HDMI (High Definition Multimedia Interface) Port:-The HDMI output is used to plug into HDTVs or monitor.

Ethernet port:-The Ethernet port connects raspberry Pi to the internet.It also plays a role in getting new software easier.

Audio output:-The audio output can be used to plug into an audio docking station. Here analog RCA connection is used.

GPIO headers:-The GPIO headers connect the Raspberry Pi to other hardware device. It will allow us to control and interact with real world.

RCA video output:-The video output is used to connect to an older type television and is connected to display if HDMI output is not used.

SD Card Slot:-The total memory of the SD card is about 8GB.

\section{ARDUINO UNO}

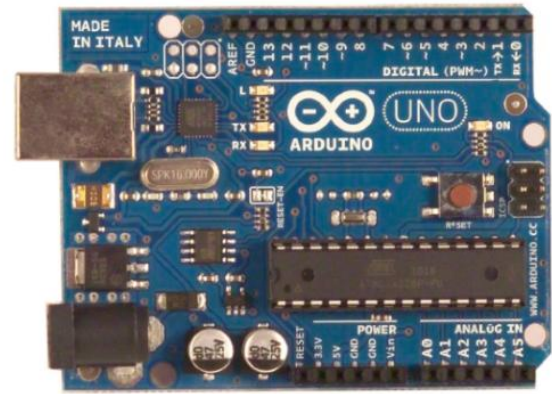




\section{IJIREEICE \\ International Journal of Innovative Research in Electrical, Electronics, Instrumentation and Control Engineering \\ ISO 3297:2007 Certified \\ Vol. 5, Issue 6, June 2017}

The Arduino Uno is a microcontroller board of ATmega328. It has 14 digital input/output pins of which 6 can be used as PWM outputs, 6 analog inputs, a USB connection, a $16 \mathrm{MHz}$ crystal oscillator, an ICSP header, reset button and a power jack. It supports the microcontroller by connecting it to a computer with a USB cable or power it with a battery to get started.

Power:-The board can operate on an external supply of 6 to $20 \mathrm{~V}$.

VIN: Input voltage to Arduino using external power supply.

$5 \mathrm{~V}$ : The regulated power supply used to power the microcontroller and other components on the board.

3V3: A 3.3 volt supply generated by the on-board regulator.

GND: Ground pins.

Memory:-The Atmega328 has $2 \mathrm{~KB}$ of SRAM and $1 \mathrm{~KB}$ of EEPROM which can be read and written with the EEPROM library. It also has $32 \mathrm{~KB}$ of flash memory for storing code of which $0.5 \mathrm{~KB}$ is used for the boot loader.

Input and Output:- It has 14 digital pins for input and output.

Serial: $0(\mathrm{RX})$ and $1(\mathrm{TX})$ : RX to receive and TX to transmit TTL serial data.

External Interrupts: 2 and 3: These pins can be configured to trigger an interrupt.

PWM: 3, 5, 6, 9, 10, and 11: Provide 8-bit PWM output.

SPI: 10(SS), 11(MOSI), 12 (MISO), 13 (SCK): These pins support SPI communication.

LED: 13: When the pin is HIGH value, the LED is on, when the pin is LOW, LED is off.

It has 6 analog inputs.

I2C: 4 (SDA) and 5 (SCL): Support I2C i.e. TWI communication.

AREF: Reference voltage for the analog inputs.

Reset: A reset button to shields which block the one on the board.

\section{BLUETOOTH MODULE}

Bluetooth Module can be used in a Master or Slave configuration, used in wireless communication. The Bluetooth Module has 6 pins VCC, GND, TX, RX, Key and LED. Bluetooth is a wireless technology standard for exchanging data over a short distance in a very efficient way. Bluetooth operates at frequencies between 2402 and $2480 \mathrm{MHz}$, or 2400 and $2483.5 \mathrm{MHz}$ including guard bands $2 \mathrm{MHz}$ wide at the bottom end and 3.5 MHz wide at top.



1. STATE:-When the module is not connected toother Bluetooth device, signal goes low. At this low state, the led flashes continuously which denotes that the module is not paired with other device.

2. RX:-TX of microcontroller.

3. TX: - RX of microcontroller.

4. GND: - Ground.

5. VCC: $-+5 \mathrm{~V}$.

6. EN:-With the help of AT commands, the user can change the parameters of this module but only when the module is not paired with any other Bluetooth device. To enable AT command mode, press the button switch for a second.

\section{USB CAMERA}

Webcam setup consists of a digital camera attached to the computer, typically through the USB port. Here the system will continuously capture video frames using wireless camera. So that target will be easily detected and we can fire at that direction automatically.

Frame rate:-A webcam will have at least 30 frames per second frame.

Resolution:-Many webcams now have 720p and 1080p high-definition capabilities.

\section{MOTOR DRIVER}

It is an electronic device which enables a voltage to be applied across a load in either direction. It allows a circuit to control full over a standard electric DC motor. With a microcontroller command the motor to go forward, reverse, brake, and coast. Each motor driver can control the speed and direction of two DC motor. 


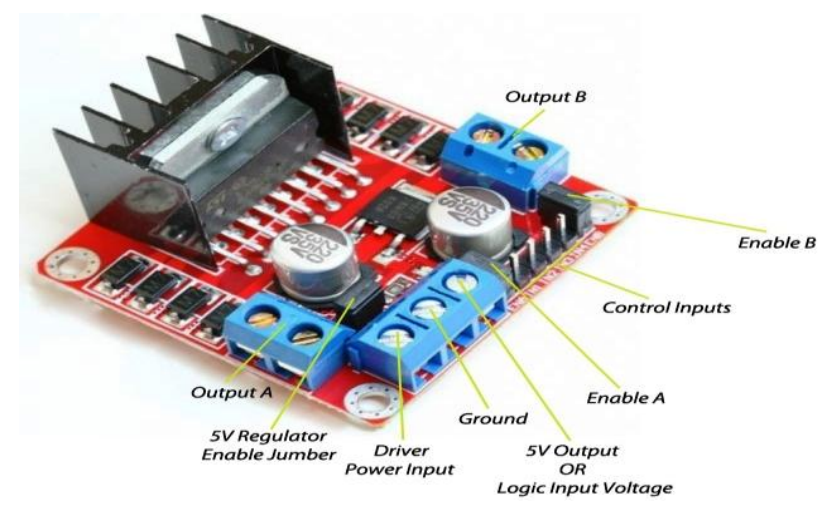

\section{MOTOR}

A DC motor is an electronic device that converts direct current power into mechanical power. The most common types depends on the forces produced by magnetic fields. Nearly all types of DC motors have some internal mechanism, eitherelectromechanical or electronic. Most types produce rotary motion whereas a linear motor directly produces force and motion in a straight line.

Here the DC motor is of 60rpm which is controlled by $12 \mathrm{~V}$ rechargeable battery.

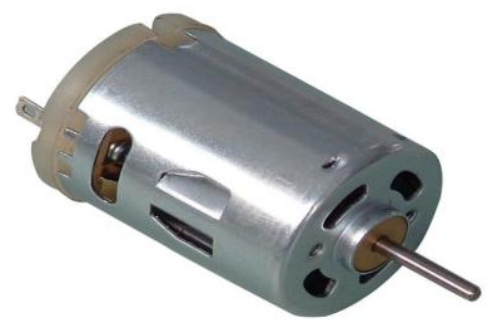

\section{SENSORS}

Smoke sensor:-It works on the light obscuration detection principle. It employs a light source and a photo sensitivity receiving device such as a photo diode. When smoke particles partially block the light beam, the reduction in light is reaching photo sensitive device alters its output. The change in output is sensed by the detector circuitry and when the threshold is closed, an alarm is initiated. The circuit uses a 555 timers and sensor and the sensor module activates the oscillator and produces an alarm.

Metal detector sensor:-A metal detector detects the presence of metal nearby. Metal detectors are useful for finding metal hidden within objects, or metal objects buried underground.

The metal detector consists of an oscillator producing an alternating current that passes through a coil producing an alternating magnetic field. If a piece of electrically conductive metal is close to the coil, eddy currents will be generated in the metal, and this produces a magnetic field of its own. If another coil is used to measure the magnetic field, the change in the field due to the metallic object can be detected.

Heat sensor:-Intension is to switch on a buzzer and an LED when temperature at the thermistor crosses a limit. The thermistor is of negative temperature coefficients type. After heating the thermistor its resistance decreases the voltage at junction of thermistor and resistor drops. Suppose after heating the thermistor which is $10 \mathrm{~K}$ ohms at room temperature, its resistance value become $9 \mathrm{~K}$ ohms. Then voltage across the thermistor reduces.

\section{WORKING}

Here in this robot the motor is inserted into chassis accordingly and screwed. The motors are fixed to the chassis using the side screwing available in the chassis. Body is placed on top of the chassis. The wheel is connected to the shaft using the screws. After connecting the motors to the chassis, checking the direction of all motors. After checking, connect the motor individually to the battery and check the direction. Here we use 8 DC motors, 4 motor is connected at the base of the robot for the movement of the wheels, rest 3motors are connected at the center of the robot for the rotation of the upper part of body of the robot and one motor is for the triggering of the gun.

The DC motor is operated at 60rpm and $12 \mathrm{~V}$ rechargeable power supply is applied which is connected to the DC motor. 


\section{IJIREEICE

Vol. 5, Issue 6, June 2017

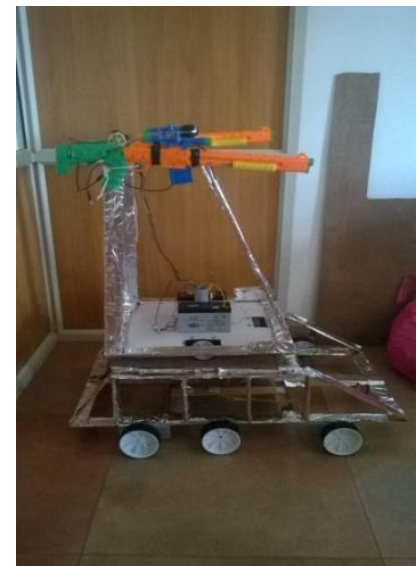

Fig. 2. Photograph of the proposed system.

Arduino and Bluetooth module are interfaced with each other. We use C programming to interface with each other. Motor driver is used, to control the motor which is connected to the Arduino. Gun is placed on top of the body of the robot of both the sides. The triggering part of the gun is connected to the battery which will trigger the gun automatically according to the instruction. Since the gun and camera is connected to the top of the body of robot, when rotates then the gun can point at any direction, even the live video streaming is done at any angle where rotation is controlled by the controller.

For live video streaming the Raspberry Pi is connected to the USB camera with the help of USB port. In the PC, Raspbian operating system is installed. Putty configuration and also VNC viewer are needed to install Raspbian OS. Virtual network computing is a Graphical desktop sharing system that allows us to remotely control the desktop interface of one computer from another. Putty configuration has SSH and Telnet client .It is open source software that is available with source code.

For the sensors the 3 colored LED lights are place in such a way that the controller can view the color of the light through camera.

\section{FLOW CHART}

The Fig. 2(a) shows the working flowchart of the proposed module. When the module is initialized it will check whether intruder is detected through camera.

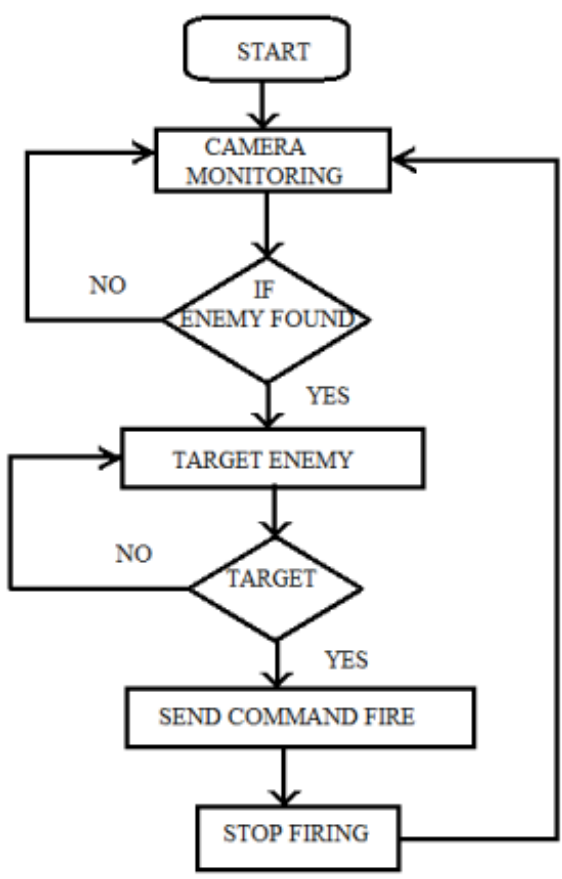

Fig. 2(a).Flowchart of the proposed system for gun triggering 


\section{IJIREEICE \\ International Journal of Innovative Research in Electrical, Electronics, Instrumentation and Control Engineering \\ ISO 3297:2007 Certified \\ Vol. 5, Issue 6, June 2017}

Fig. 2(b) shows the flow diagram of sensing unit. When the module is initialised it will check whether smoke is sensed, metal is detected or heat is detected. If detected particular LED will be ON and that light is viewed on the camera.

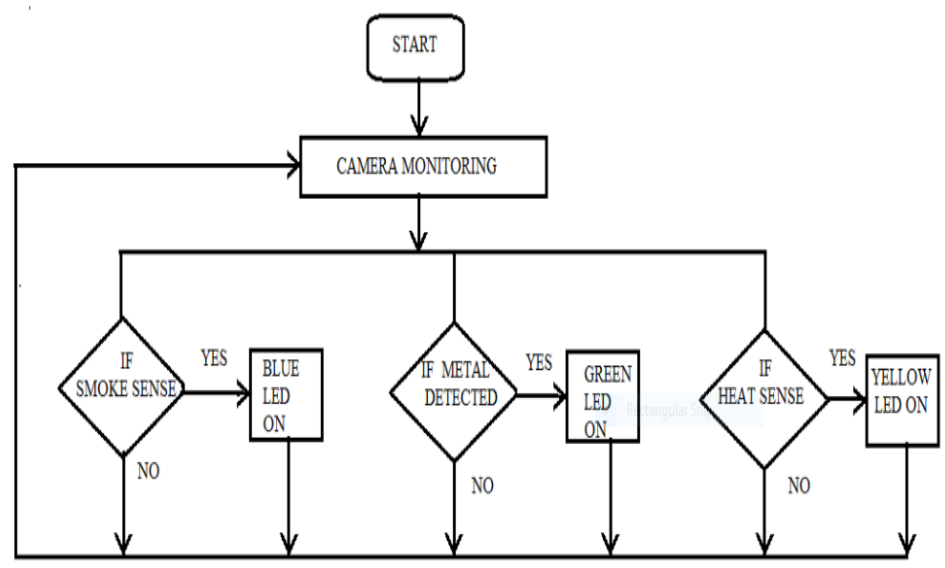

Fig. 2(b).Flowchart of sensing unit

\section{IV.RESULTS AND ANALYSIS}

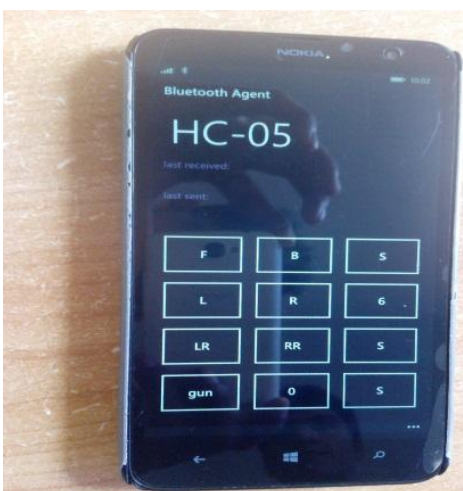

Fig. 3.1. Sending command from Bluetooth to robot unit

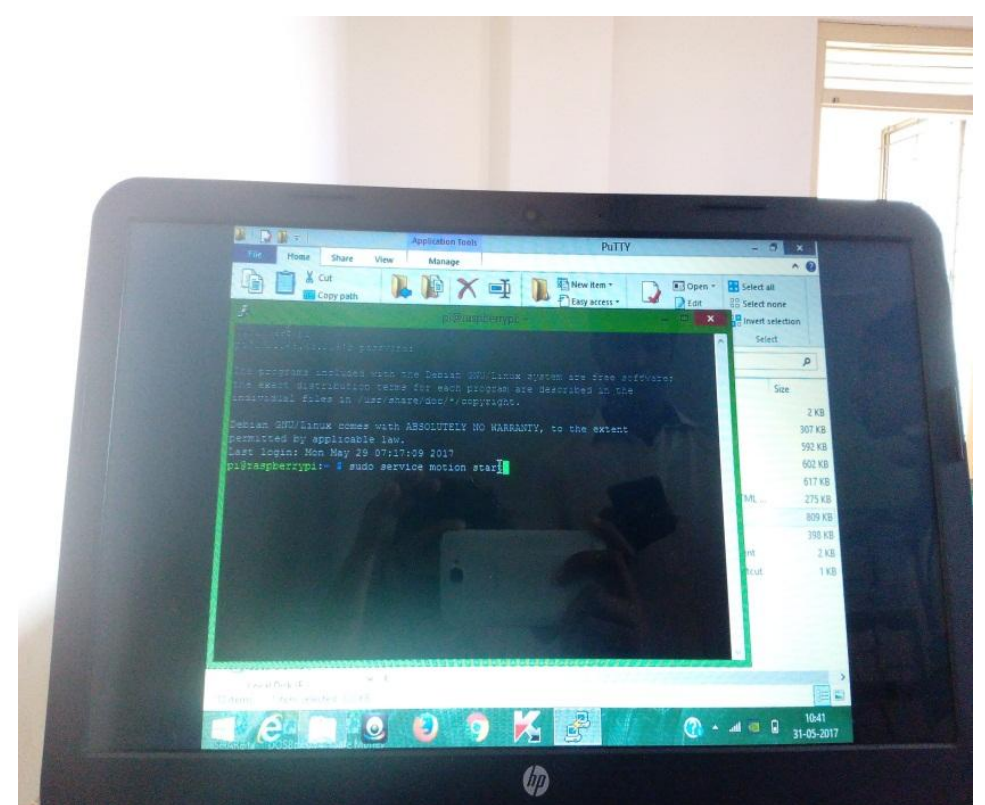

Fig. 3.2.Sending command to Raspberry pi using putty software. 


\section{IJIREEICE \\ ISO 3297:2007 Certified \\ Vol. 5, Issue 6, June 2017}

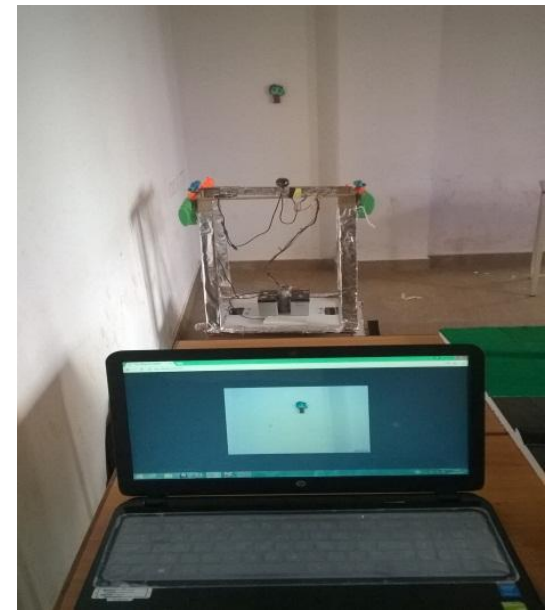

Fig. 3.3. Target live video stream viewed in laptop.

From Fig. 3.1 using Bluetooth sending command to robot unit to move front, back, right and left. Also sending command to trigger the gun, to rotate the upper part of robot clockwise or anticlockwise.

Fig. 3.2 shows sending command to Raspberry pi module using putty software for the live video streaming; In Fig. 3.3 we can see the target is viewed in laptop due to live video streaming using Raspberry pi.

\section{CONCLUSION}

The robot can send live video streaming wirelessly to the controller using camera and Raspberry pi interface. If the enemy is found, he is targeted by triggering the gun using Bluetooth Arduino technology. The camera and the gun are connected to upper part of the body, so the rotation is $360^{\circ}$ i.e. the camera can video stream in all direction as well as the gun can point at all the direction. The sensors will detect the smoke, metal or if there is any fire by viewing the LED light rays emitted just below the camera.

\section{ACKNOWLEDGMENT}

We are thankful to Management of Shri Madhwa Vadiraja Institute of Technology and Management and all our faculty members of E\&C Department for their encouragement, support and whole hearted cooperation. We would also like to thank Associate Prof. Nagaraja Rao for his guidance and valuable suggestions time to time that helped us to complete our work.

\section{REFERENCES}

[1] M.S999999aitoh, Y.Takahashi, A.Sankaranarayanan, H.Ohmachi, K.Marukawa, “A Mobile Robot Testbed with Manipulator for a Security Guard Application”, SECOM Intelligent Systems Lab, Sakae, Tachikawa, Tokyo, Japan, 1995.

[2] Goris, K, “Autonomous Mobile Robot Mechanical Design”, Vrije Universiteit Brussel, Brussels, Belgium, 2005.

[3] Shakhatreh, F, "The Basics of Robotic", Lahti University, Syksy, Finland, 2011.

[4] Paul E. Sandin, "Robot Mechanisms and Mechanical Devices", The McGraw-Hill Companies USA, 2003. 